livraisons

d'Histoire

de l'Architecture

\section{Livraisons de l'histoire de l'architecture}

$20 \mid 2010$

Ceci tuera cela

\title{
Quoi tuera quoi ? Les enjeux de l'invention de l'imprimerie chez Victor Hugo et Gérard de Nerval
}

What will kill what? Hugo and Nerval: The challenges born by the invention of the print

Was wird was töten? Zielsetzungen der Erfindung des Buchdrucks bei Hugo und Nerval

Max Milner (

\section{OpenEdition}

Journals

Édition électronique

URL : http://journals.openedition.org//ha/251

DOI : $10.4000 /$ lha. 251

ISSN : 1960-5994

Éditeur

Association Livraisons d'histoire de l'architecture - LHA

Édition imprimée

Date de publication : 10 décembre 2010

Pagination : 9-18

ISSN : 1627-4970

Référence électronique

Max Milner (†), «Quoi tuera quoi ? Les enjeux de l'invention de l'imprimerie chez Victor Hugo et Gérard de Nerval », Livraisons de l'histoire de l'architecture [En ligne], 20 | 2010, mis en ligne le 10 décembre 2012, consulté le 01 février 2020. URL : http://journals.openedition.org//ha/251 ; DOI : 10.4000/lha. 251

Ce document a été généré automatiquement le 1 février 2020

Tous droits réservés à l'Association LHA 


\section{Quoi tuera quoi ? Les enjeux de l'invention de l'imprimerie chez Victor Hugo et Gérard de Nerval}

What will kill what? Hugo and Nerval: The challenges born by the invention of the print

Was wird was töten? Zielsetzungen der Erfindung des Buchdrucks bei Hugo und Nerval

Max Milner ( $\dagger$ )

1 La question de l'invention de l'imprimerie a engendré une abondante littérature tout au long du XIX ${ }^{\mathrm{e}}$ siècle. Mais celle-ci a surtout porté sur l'histoire des dispositifs techniques qui l'ont rendue possible ou ont favorisé son développement, et, surtout à une époque où l'orgueil national infiltrait les causes les plus variées, sur la priorité qu'il convenait d'accorder à tel ou tel pays revendiquant l'honneur d'avoir donné naissance ou asile à l'un de ses inventeurs putatifs. L'année 1840, où fut inaugurée en grande pompe la statue de Gutenberg à Strasbourg, fut particulièrement féconde à cet égard. En revanche, on trouve peu de réflexions sur les modifications que l'imprimerie apporta à la nature et au régime de la communication entre les hommes, en dehors d'une quasi universelle célébration de ses bienfaits. Il est d'autant plus frappant de voir Victor Hugo, dès 1830, accompagner cette célébration d'une extraordinaire "vue à vol d'oiseau " portant sur la diffusion et sur la nature même de la pensée humaine des origines à nos jours. Malgré les simplifications et les erreurs que comporte une telle entreprise, les perspectives qu'ouvrent les chapitres 1 et 2 du livre V de Notre-Dame de Paris contiennent des intuitions dont je vais essayer de préciser l'importance pour l'avenir de ce que nous appelons les médias. Celles que Gérard de Nerval développe une vingtaine d'années plus tard dans L'Imagier de Harlem sont d'une tout autre nature, mais elles permettent de poser, sur le rôle politique et social de l'imprimerie, une question dont la réponse n'est pas aussi simple qu'il y paraît. Sans nier l'arbitraire du 
rapprochement, je pense qu'il n'est pas sans intérêt de prendre en considération ces deux textes.

2 «Ceci tuera cela. » À quoi bon, dira-t-on, s'interroger sur le sens de la prophétie de Claude Frollo, puisque le romancier se charge lui-même d'élucider «la pensée qui se dérobait sous ces paroles énigmatiques »? En réalité les choses ne sont pas si simples. D'abord parce qu'il y a là, nous dit Hugo, non pas une, mais deux pensées. La première, relativement claire, peut se traduire par "l'imprimerie tuera l'édifice», et correspond en gros au discours libéral sur l'opposition entre le pouvoir de l'Église et le progrès des lumières, dont l'imprimerie a été le principal agent. Dans la bouche de l'archidiacre de Notre-Dame, la phrase ne peut signifier qu'une crainte et un regret. On sait de reste - et la fin du texte le confirmera - qu'à l'époque où il l'écrit, Victor Hugo, qui n'est plus le défenseur patenté du trône et de l'autel, ne peut lui donner qu'un sens positif.

Il en va tout autrement de la deuxième "pensée » contenue dans la phrase célèbre pensée que Victor Hugo traduit par la formule suivante: "L'imprimerie tuera l'architecture. » Comment imaginer que celui qui avait écrit une Note sur la destruction des monuments en France, jetée dans le débat public en 1829 et reprise en 1832, puis en 1834 sous le titre Guerre aux démolisseurs! ait pu considérer la disparition de l'architecture, même au profit de l'imprimerie, comme un bien pour la civilisation ? Si cette défense des monuments de l'ancienne France a pu être partiellement inspirée, au début, par le culte du passé qui régnait dans les milieux ultras (et par l'appartenance des « démolisseurs » à une bourgeoisie mercantile), le reste de la vie et de l'œuvre de Victor Hugo (écrite et dessinée) montre que son goût de l'architecture médiévale est une constante de son esthétique qui touche à la fascination. Se borner à enregistrer une contradiction, chez un penseur qui connut d'autres partages, entre l'amour des vieilles pierres et l'appel du futur serait se condamner à ignorer la portée profonde du texte que nous tentons d'interpréter.

4 Peut-être faudrait-il commencer par dissiper une équivoque concernant la notion même d'architecture. Dans quel sens faut-il entendre que l'architecture, « seule entre tous les arts », n'a « plus d'avenir ${ }^{1}$ » - ce qui paraitt corroborer purement et simplement la prophétie de Claude Frollo? Cette affirmation doit évidemment être lue dans le contexte de la polémique ouverte par Guerre aux démolisseurs! Celle-ci vise non seulement les démolitions pures et simples, destinées à récupérer des matériaux, mais aussi et surtout celles qui ont pour but de remplacer des monuments médiévaux par des édifices modernes. De là les sarcasmes contre « les trois ou quatre églises cubiques que vous bâtissez piteusement çà et là ", ou contre " votre Madeleine, ce tome deux de la Bourse, avec son lourd tympan qui écrase sa maigre colonnade ${ }^{2}$ ». Il s'agit dans tous les cas d'édifices construits dans le style néoclassique, dont les partisans sont mis sans ménagements dans le même sac que les adversaires du romantisme, dont Victor Hugo estime, avec quelque optimisme, que la cause est définitivement perdue :

«Le ridicule duel des classiques et des romantiques s'est arrangé de lui-même, tout le monde étant à la fin du même avis [ceci écrit en 1829 !]. Il n'y a plus de question. [...] À peine y a-t-il encore, dans l'arrière-parloir des collèges, dans la pénombre des académies, quelques bons vieux enfants qui font joujou dans leur coin avec les poétiques et les méthodes d'un autre âge; qui poètes, qui architectes; celui-ci s'ébattant avec les trois unités, celui-là avec les cinq ordres; les uns gâchant du plâtre selon Vignole, les autres gâchant des vers selon Boileau ${ }^{3}$. »

5 Si le parallèle doit être pris au pied de la lettre, il conduit à des conclusions inquiétantes. L'avenir de la littérature, c'est le romantisme et ce qui viendra ensuite. 
Quant à l'avenir de l'architecture, c'est... l'architecture médiévale (surtout celle du gothique flamboyant), conservée du mieux que possible. Est-ce la faute des architectes modernes et de leur manque de goût et d'imagination? Certes, Victor Hugo n'est pas tendre envers eux. Mais il y a plus grave : c'est que l'architecture semble avoir pour lui un lien intrinsèque avec le passé, parce qu'elle n'est pas ce que nous désignons actuellement par ce nom, c'est-à-dire un art de bâtir variable selon les époques, mais avant tout un langage, et un langage symbolique, dont le fonctionnement est inadapté aux besoins des époques à venir. L'intérêt du chapitre «Ceci tuera cela » réside moins dans les vues discutables sur le passé que dans ce qu'il dessine en ce qui concerne l'avenir de la communication, et notamment de la communication de masse.

6 La manière dont Victor Hugo développe sa thèse suppose en fait deux conceptions du symbole dont la juxtaposition justifiera à la fois la continuité supposée entre l'édifice et le livre et l'importance de la rupture que constitue l'invention de l'imprimerie. Cette juxtaposition, qui est en même temps une imbrication, peut être facilement repérée dans le paragraphe suivant:

"L'idée mère, le verbe, n'était pas seulement au fond de tous ces édifices, mais encore dans la forme. Le temple de Salomon, par exemple, n'était point seulement la reliure du livre saint, il était le livre saint lui-même. Sur chacune de ses enceintes concentriques les prêtres pouvaient lire le verbe traduit et manifesté aux yeux, et ils suivaient ainsi ses transformations de sanctuaire en sanctuaire jusqu'à ce qu'ils le saisissent dans son dernier tabernacle sous sa forme la plus concrète qui était encore de l'architecture : l'arche. Ainsi le verbe était enfermé dans l'édifice mais son image était sur son enveloppe comme la figure humaine sur le cercueil d'une momie $^{4}$.»

7 Toute l'équivoque plus ou moins consciemment entretenue par Victor Hugo tourne autour de la préposition «sur ». Que la structure concentrique du temple de Jérusalem ait eu une signification propre, pouvant être qualifiée à la rigueur de symbolique, que les croyants ou les prêtres, en franchissant ses différentes enceintes jusqu'au Saint des Saints, aient eu à accomplir un parcours initiatique signifiant l'extrême secret qui protège la parole divine, c'est là une forme de symbolisme qu'on attache volontiers aux dispositions architecturales depuis la plus haute antiquité jusqu'à nos jours. Mais en écrivant que les prêtres pouvaient lire sur chacune des enceintes concentriques «le verbe traduit et manifesté aux yeux» et "suivaient ainsi ses transformations de sanctuaire en sanctuaire", Hugo suggère une idée illustrée par un grand nombre d'exemples, avant et après ce texte : c'est que le langage de l'édifice ne se limite pas au symbole qu'il constitue en lui-même, à la "phrase ", si l'on veut (il vaudrait mieux parler de signe ou d'emblème), qu'il inscrit dans l'espace, mais qu'il s'étend aux symboles de pierre ou gravés dans la pierre qui prolifèrent, de plus en plus nombreux avec le temps, à l'extérieur ou à l'intérieur de ses murs. C'est ainsi que, le symbole se multipliant et se diversifiant à proportion du développement de la pensée humaine, se sont construits «ces livres mystérieux qui étaient aussi de merveilleux édifices: la pagode d'Eklinga, le Rhamseion d'Égypte, le temple de Salomon ${ }^{5}$ ».

8 Toute l'évolution de l'architecture qui est retracée ensuite, en une vue merveilleusement cavalière (dans les deux sens du terme), dépend en somme de cette superposition entre le symbole proprement architectural que constitue l'édifice luimême et le symbolisme didactique qui se développe en lui ou autour de lui. L'un et l'autre contribuent à créer des formes qui expriment l'esprit d'une époque : formes figées, oppressantes, mystérieuses dans les âges que Victor Hugo qualifie de 
théocratiques : «Ce sont des livres ténébreux que les initiés seuls savent déchiffrer [...]. Ne demandez pas aux maçonneries hindoue, égyptienne, romane qu'elles réforment leur dessin ou améliorent leur statuaire. Tout perfectionnement leur est impiété. Formes plus aérées, souples, dociles à l'esprit du temps, facilement intelligibles, lorsque s'allège l'emprise des prêtres et du dogme et que le peuple participe à leur élaboration.

On voit l'évolution sémantique du mot «symbole " quand on passe de l'une à l'autre phase. Dans la première, le rapport de signifiant à signifié était d'autant plus immuable qu'inscrit dans la pierre d'une façon indélébile : « Dans ces architectures, il semble que la roideur du dogme se soit répandue sur la pierre comme une seconde pétrification ${ }^{7}$. » Dans la seconde phase se produit un allègement, une autonomisation relative du signifiant qui fait que l'emploi du mot symbole, dans le sens qui avait été précédemment le sien, devient quelque peu problématique. Les architectures qui se manifestent alors « ont quelque chose d'humain qu'elles mêlent sans cesse au symbole divin sous lequel elles se produisent encore. De là des édifices pénétrables à toute âme, à toute intelligence, à toute imagination, symboliques encore, mais faciles à comprendre comme la nature ${ }^{8}$.»

Quelle est, dans ces conditions, non seulement le véritable progrès, mais la véritable mutation qu'introduit l'invention de l'imprimerie? C'est incontestablement le divorce entre la pensée et son support. Il y a, à vrai dire, une étape sur laquelle Victor Hugo passe assez allègrement, c'est celle du manuscrit, et même, en remontant plus haut, celle de l'écriture syllabique, véritable médiateur entre le symbolisme compact de l'idéogramme, de l'image sculptée ou du monument, et le symbolisme labile de la phrase et du texte ${ }^{9}$. Mais l'allègement qu'introduisent le manuscrit et sa relative reproductibilité se paient d'un prix très lourd, qui est sa fragilité et sa rareté. $\mathrm{Ne}$ reculant pas devant le paradoxe, Hugo avance, avec une certaine vraisemblance, que si l'édifice l'emporte en solidité sur le manuscrit, le livre imprimé l'emporte sur l'édifice par la faculté que lui donne son indéfinie multiplication de surmonter tous les aléas de la durée : «Au quinzième siècle tout change. La pensée humaine découvre un moyen de se perpétuer non seulement plus durable et plus résistant que l'architecture, mais encore plus simple et plus facile ${ }^{10}$. »

11 Il faut insister sur cette facilité et cette simplicité, car là se trouve sans doute le cœur de l'intuition hugolienne. Il ne s'agit pas seulement, en effet, d'une commodité pratique, d'une plus grande aisance dans la reproduction et la diffusion d'une pensée qui demeurerait identique à ce qu'elle était auparavant. C'est le régime même de la pensée, c'est son mode de fonctionnement et pour ainsi dire son essence qui se trouvent radicalement transformés par l'invention de l'imprimerie: "C'est la révolution mère. C'est le mode d'expression de l'humanité qui se renouvelle totalement, c'est la pensée humaine qui dépouille une forme et en revêt une autre ${ }^{11}$. " En effet, dans la mesure où le symbole était partie intégrante du monument, il conservait, même relativement libéré de ses attaches théocratiques, un caractère massif qui faisait obstacle au libre développement de la pensée. En cassant le symbole, en donnant à ses éléments constitutifs leur autonomie, l'imprimerie offrait à ces éléments la possibilité de réemplois infinis selon les besoins des époques successives, qui jalonnent - Victor Hugo n'en doute pas - les progrès de l'humanité. Cela, encore une fois, non seulement grâce à la multiplication matérielle du livre, mais aussi, en définitive, à cause de la souplesse d'un procédé - la mobilité des caractères et des mots - qui substitue à la copie servile du moine les variations introduites par l'inventeur ou 
le poète ${ }^{12}$. Cet allègement, gage de durée, mais aussi de vivacité, d'audace, d'inventivité, Hugo le traduit par une série de métaphores dont on s'en voudrait d'alourdir par un commentaire la puissance d'évocation :

«Sous la forme imprimerie, la pensée est plus impérissable que jamais; elle est volatile, insaisissable, indestructible. Elle se mêle à l'air. Du temps de l'architecture, elle se faisait montagne et s'emparait puissamment d'un siècle et d'un lieu. Maintenant elle se fait troupe d'oiseaux, s'éparpille aux quatre vents, et occupe à la fois tous les points de l'air et de l'espace.

Nous le répétons, qui ne voit que de cette façon elle est bien plus indélébile? De solide qu'elle était elle devient vivace. Elle passe de la durée à l'immortalité. On peut démolir une masse, comment extirper l'ubiquité ? Vienne un déluge, la montagne aura disparu depuis longtemps sous les flots que les oiseaux voleront encore; et qu'une seule arche flotte à la surface du cataclysme, ils s'y poseront, surnageront avec elle, assisteront avec elle à la décrue des eaux, et le nouveau monde qui sortira de ce chaos verra en s'éveillant planer au-dessus de lui, ailée et vivante, la pensée du monde englouti ${ }^{13}$.»

12 Ces images d'une légèreté qui défie le temps et l'espace sont encore dans son esprit lorsque, vingt-cinq ans plus tard, Hugo dédie « à celle qui est restée en France » le livre des Contemplations auquel le poème qui porte ce titre sert d'épilogue :

"Ce livre, légion tournoyante et sans nombre

D'oiseaux blancs dans l'aurore et d'oiseaux noirs dans l'ombre

$[\ldots]$

Prends ce livre et fais-en sortir un divin psaume!

Qu'entre tes vagues mains il devienne fantôme!

Qu'il blanchisse, pareil à l'aube qui pâlit,

À mesure que l'œil de mon ange le lit,

Et qu'il s'évanouisse, et flotte et disparaisse

Ainsi qu'un âtre obscur qu'un souffle errant caresse,

Ainsi qu'une lueur qu'on voit passer le soir,

Ainsi qu'un tourbillon de feu de l'encensoir,

Et que, sous ton regard éblouissant et sombre,

Chaque page s'en aille en étoiles dans l'ombre ${ }^{14}$."

13 Que cette évanescence, qui tient au pouvoir de dissémination du livre, soit ici l'objet d'un vœu fervent, on le comprend, dans la mesure où Hugo s'adresse à une morte qui, bien que murée dans son tombeau, communique avec l'espace infini. Il va de soi, pourtant, que la multiplication du texte imprimé n'est souhaitable que si elle n'aboutit pas à son annulation. Il est un passage de Quatre-vingt treize dont Victor Brombert a bien senti la valeur de parabole. La scène se passe dans la Tourgue assiégée, où se sont réfugiés trois petits enfants de vingt mois à quatre ans. Ils ont par jeu mis en pièces un volume précieux contenant l'évangile apocryphe de saint Barthélemy et en jettent les morceaux par la fenêtre :

« Page à page, émietté par ces petits doigts acharnés, presque tout l'antique livre s'envola dans le vent. Georgette [la plus petite], pensive, regarda ces essaims de petits papiers blancs se disperser à tous les souffles de l'air et dit :

- Papillons.

Et le massacre se termina par un évanouissement dans l'azur ${ }^{15}$. »

Papillons, oiseaux... Le "massacre de Saint Barthélemy» perpétré par des mains innocentes n'a, bien entendu, rien à voir avec l'envol des livres par delà les océans et les déluges; c'en est même, en un sens, le contraire burlesque, puisqu'il s'agit ici de détruire et là de construire. Mais la parfaite superposition des images révèle une inquiétude relative à la volubilité si vantée de l'imprimé. On ne peut se réjouir de ce 
que ceci tue cela que si le livre, au lieu de s'éparpiller aux quatre vents de l'esprit, forme à son tour une architecture aussi solide que la cathédrale, et c'est là tout le problème, sur lequel la réflexion de Victor Hugo sur son œuvre revient de façon obsédante. Dans la collection de pensées éparses qu'il avait, semble-t-il, l'intention de publier sous le titre de Tas de pierres, on trouve, datant des environs de 1830, ces deux réflexions, inspirées par ce titre même et où s'expriment successivement la hantise de l'émiettement et l'espoir d'un prodige architectural. La première se lit comme suit :

«Le tas de pierres

Préface

Qu'y a-t-il dans ce livre?

Est-ce du passé?

Est-ce de l'avenir?

Le lecteur jugera si ce tas de pierres provient d'un monument en ruine ou d'un édifice en construction ${ }^{16}$.

15 La seconde réflexion vient à la suite de l'«inexprimable admiration » qu'il dit avoir éprouvée en méditant sur le travail prodigieux de l'architecte de Notre-Dame de Paris :

«Mais c'est plus que de l'admiration, c'est un étonnement mêlé de religieuse adoration pour mon créateur quand, à l'aide de cet œil intérieur qu'on nomme la rêverie, je vois au-dedans de moi, fourmillement immense et tumultueux, ces autres ouvriers merveilleux, les pensées, qui avec des mots bâtissent une cathédrale dans mon esprit ${ }^{17}$. »

16 Cependant, ce n'est pas l'image d'une cathédrale qui lui vient, à la fin du chapitre «Ceci tuera cela", pour symboliser l'édifice que construisent, grâce à l'imprimerie, les générations successives. Une cathédrale, même se transformant de siècle en siècle, suppose une idée d'unité et d'achèvement qui ne conviendrait pas ici : «Cet édifice est colossal [...] C'est la fourmilière du genre humain. C'est la ruche où toutes les imaginations, ces abeilles dorées, arrivent avec leur miel. L'édifice a mille étages [...]» On voit où va la métaphore : "C'est la seconde tour de Babel du genre humain ${ }^{18}$."

Or cette image, qui revient souvent dans l'œuvre de Victor Hugo, est loin de n'avoir que des connotations positives. Dans Les Feuilles d'automne elle est au centre d'un véritable cauchemar :
«Mille ouvriers humains, laissant partout leurs traces,
Travaillaient nuit et jour, montant, croisant leurs pas,
Parlant chacun leur langue et ne s'entendant pas;
Et moi je parcourais, cherchant qui me réponde,
De degrés en degrés cette Babel du monde ${ }^{19}$.»

18 Si la « Babel démesurée » qu'il croit, dans le même recueil, « voir jusqu'au ciel monter, monter toujours» en contemplant les nuages le fascine par son mouvement ascensionnel, c'est "l'œil épouvanté » qu'il porte sur elle ses regards ${ }^{20}$. Considérées rétrospectivement dans Les Rayons et les Ombres («Puits de l'Inde »), les « effrayantes Babels que rêvait Piranèse » deviennent le gouffre dont les écroulements symbolisent tous les échecs de l'histoire humaine, et « la Vision d'où est sorti ce livre », qui servira de prologue à la seconde série de La Légende des siècles, reste fidèle à l'idée de cette «Babel en creux » dont parle Michel Butor ${ }^{21}$ :

«C'est l'incommensurable et tragique monceau

Où glissent, dans la brèche horrible, les vipères

Et les dragons, avant de rentrer aux repaires,

Et la nuée avant de remonter au ciel ;

Ce livre, c'est le reste effrayant de Babel ${ }^{22}$. » 
19 Entre le symbolisme compact, infrangible, fatalement enkysté dans le passé de la cathédrale et les Babels problématiques dont l'invention de l'imprimerie permet de rêver, faut-il choisir? La grandeur de Victor Hugo, d'où résulte la fécondité de son œuvre, aura été non pas de refuser le choix (la première solution étant évidemment exclue, malgré son admiration pour l'architecture médiévale), mais de préférer, dans l'édification de sa propre Babel, le mouvement ininterrompu de la création poétique au délire monumental des Babels passées, dont les décombres jonchent les gouffres de l'histoire, malgré l'aspiration qu'il lui arrive de confesser à «se totaliser en un livre complet $^{23}$ ». Défaisant les symbolismes du présent et de l'avenir, y compris les siens propres, à mesure qu'ils ont tendance à s'ériger en monuments, travaillant à devenir cet « Hugo fantôme », dont a si bien parlé Pierre Albouy ${ }^{24}$. Fidèle en cela aux leçons qu'il tirait de l'invention de l'imprimerie? Certes, et plus encore qu'il ne le prévoyait en écrivant "Ceci tuera cela ». Jusqu'où ira l'accomplissement de sa prophétie, avec les contradictions et les déchirements de conscience qu'elle implique, non seulement dans l'avenir de son œuvre, mais dans celui de la communication humaine? Rêver d'une pensée indépendante de son support ne va pas sans renoncements et sans pertes. Le livre aussi est un support, moins rigide que la pierre, mais qui comporte aussi sa propre symbolique, magnifiquement explorée par Mallarmé. Et la fin de la "galaxie Gutenberg » est loin d'avoir aboli les conglomérats de symboles dans lesquels vient se prendre la liberté de la pensée. On peut même dire qu'elle les a rendus plus redoutables, du fait qu'il est plus difficile de détecter où et comment ils se constituent. "Ceci tuera cela» n'est pas seulement une prophétie qu'il faut se réjouir de voir accomplie. C'est aussi un appel à la vigilance, que le développement futur de l'œuvre de Victor Hugo nous permet d'entendre.

Je voudrais pour finir parler de Gérard de Nerval. Je me contenterai de dire pourquoi j'avais eu l'idée de l'évoquer à la suite - sinon en compagnie - de Victor Hugo. C'est le seul écrivain du XIX siècle qui ait consacré à l'invention de l'imprimerie une œuvre de fiction de quelque importance comportant une réflexion sur ses enjeux. Certes L'Imagier de Harlem n'est pas un chef d'œuvre - et c'est le fruit d'une collaboration dans laquelle il est impossible de savoir quelle est la part exacte de Nerval, quoique l'essentiel de l'intrigue soit à peu près certainement de lui. Mais ce drame fantastique ne se borne pas à célébrer les mérites de l'inventeur, qui est ici Coster, assisté de Gutenberg, Faust et Schoeffer. S'inspirant du Faust de Klinger, Nerval imagine que l'invention de l'imprimerie a été favorisée par le diable en vue des facilités qu'elle lui offrirait pour répandre le mal parmi les hommes. La pièce montrera comment ces espoirs ont été déjoués et se terminera par un hymne à l'imprimerie, dont le pouvoir libérateur sera salué en termes aussi lyriques - sinon aussi poétiques - que dans Notre-Dame de Paris ("Second soleil, œuvre sublime !/ Tout l'univers va vous bénir / Et chaque feuille qu'on imprime / Est un rayon pour l'avenir !»). Mais nous aurons assisté, chemin faisant, à quelques détournements de ce rôle bénéfique à travers les siècles, que le caractère fantastique de l'intrigue nous permet de parcourir. Le diable, sous un déguisement, utilise l'invention de Coster pour fabriquer de la fausse monnaie et dit à l'inventeur penaud: «Tu est déjà contrefait... » Allusion voilée au problème de la contrefaçon belge, dont Nerval se préoccupera, ainsi que plusieurs de ses confrères, mais symbole aussi des manipulations de la pensée que sa reproduction mécanique rend possibles. Ces billets de banque, justement, inspirés des assignats que Méphistophélès répand dans le second Faust, sont un premier exemple de la perversion d'un instrument destiné 
à répandre les idées, et utilisé pour multiplier l'or - et le faux... D'autres exemples suivront : un mauvais recueil de nouvelles de Louis XI, qui se vend très bien à cause du nom de l'auteur (le XXI ${ }^{e}$ siècle aurait ici des leçons à entendre), et dont le Roi se réjouit de faire commerce. Puis un pamphlet qui étrille le Roi, et avec lui le recueil en question, ce qui vaut à Coster d'être mis au cachot; les livres de sciences occultes qui se répandent dans le public; le diable, sous l'apparence de Machiavel, conseiller de César Borgia, inondant Rome d'ouvrages licencieux, s'attribuant, grâce au privilège, l'exclusivité de la production imprimée, pour le plus grand mal de l'humanité, et pouvant enfin s'écrier: "Merci, Coster; tu avais inventé une arme pour tuer Satan, Satan la saisit pour se défendre, et tu vas voir ce que je sais faire de l'arme la plus innocente, quand je l'empoisonne avec le venin de mon enfer ${ }^{25}$.»

21 Ces vues n'ont pas la profondeur de celles de Victor Hugo, mais elles méritent quand même, je crois, de figurer dans les archives de la médiologie.

\section{NOTES}

1. . "S'il est vrai, comme nous le croyons, que l'architecture, seule entre tous les arts, n'ait plus d'avenir, employez vos millions à conserver à entretenir, à éterniser les monuments nationaux et historiques qui appartiennent à l'état, et à racheter ceux qui sont aux particuliers. » (Victor Hugo, "Guerre aux démolisseurs!", Euvres complètes, Paris, Club Français du Livre, tome IV, 1967, p. 508.) Nos citations de Hugo seront toutes empruntées à cette édition.

2. . Victor Hugo, « Guerre aux démolisseurs », op. cit., tome IV, 1967, p. 507.

3. Idem.

4. . Victor Hugo, Notre-Dame de Paris, op. cit., tome IV, 1967, p. 137.

5. . Idem.

6. . Victor Hugo, Notre-Dame de Paris, op. cit., tome IV, 1967, p. 140.

7. . Idem.

8. . Idem.

9. C'est par un abus manifeste que Victor Hugo écrit, après avoir évoqué les mégalithes : «Plus tard on fit des mots. On superposa la pierre à la pierre, on accoupla ces syllabes de granit, le verbe essaya quelques combinaisons. Le dolmen et le cromlech celtes, le tumulus étrusque, le galgal hébreu sont des mots. [...] L'immense entassement de Karnac est déjà une formule tout entière. » (Victor Hugo, Notre-Dame de Paris, op. cit., tome IV, 1967, p. 137)

10. . Victor Hugo, Notre-Dame de Paris, op. cit., tome IV, 1967, p. 141.

11. Idem.

12. Sur la manière dont l'imprimerie se détache peu à peu de la copie pure et simple de la page manuscrite pour transformer le mode de présentation du texte d'une manière qui révolutionnera sa perception par le lecteur, voir Elizabeth L. Eisenstein, La Révolution de l'imprimerie à l'aube de l'époque moderne, traduction Maud Sissung et Marc Duchamp, Paris, La Découverte, 1991.

13. . Victor Hugo, Notre-Dame de Paris, op. cit., tome IV, 1967, p. 141.

14. . Victor Hugo, Contemplations, op. cit., tome IX, 1968, p. 394-397. Voir le beau commentaire de ces vers par Bernard Leuilliot, " «Ceci tuera cela": le roman et le paradoxe littéraire", Littérature, $\mathrm{n}^{\circ}$ 36, Paris, Larousse, Armand Colin, décembre 1979, p. 15.

15. . Victor Hugo, Quatre-Vingt Treize, op. cit., tome XV, 1970, p. 442. 
16. . Victor Hugo, « Le tas de pierres », op. cit., tome IV, 1967, p. 929.

17. Ibid., p. 930.

18. . Victor Hugo, Notre-Dame de Paris, op. cit., tome IV, 1967, p. 144.

19. . Victor Hugo, Les Feuilles d'automne, op. cit., tome IV, 1967, p. 428.

20. Ibid., p. 443.

21. . Michel Butor, dans les Euvres complètes de Victor Hugo déjà citées, tome VIII, 1968, p. XIXXXXII.

22. . Victor Hugo, La Légende des siècles, op. cit., tome X, 1969, p. 821.

23. . Victor Hugo, « Feuilles paginées », op. cit., tome III, 1967, p. 1195.

24. . Voir Pierre Albouy, Mythographies, José Corti, 1976, p. 260 et suiv.

25. . Gérard de Nerval, Léo Burckart. L'Imagier de Harlem, édition Jacques Bony, Flammarion, 1996, p. 406.

\section{RÉSUMÉS}

Sachant le goût de Hugo pour l'architecture médiévale, il convient de s'interroger que le sens exact du "Ceci tuera cela", qui voit la littérature s'opposer radicalement à l'architecture. Développant l'idée d'une architecture comme langage symbolique plutôt que comme «art de bâtir », Hugo porte son attention sur le support, dont les variations influent sur l'autonomie et la nature du message transmis : en passant de l'édifice au livre, le message passe de l'affirmation monolithique à un éparpillement synonyme tantôt d'immortalité, tantôt de disparition. Investissant l'ensemble de la poétique hugolienne, cette intuition de l'ubiquité de l'imprimerie rejoint une idée plus isolée de Nerval qui, dans L'Imagier de Harlem, fait de l'imprimerie un outil pouvant servir la connaissance comme la tromperie. Même si le texte de Nerval n'a pas la portée de celui de Hugo, tous deux sont parmi les premiers penseurs de ce qui sera bien plus tard appelé la médiologie.

Knowing Hugo's taste for medieval architecture, it's important to think about the exact meaning of "This will kill that" in which literature is seen as the complete opposite of architecture. By his presentation of architecture as a symbolical language rather than an "art of building things", Hugo focuses his attention on the medium whose changes affect the nature and the autonomy of the transmitted message: as the medium conveying him changes, the monolithic affirmation of the message scatters into immortality or nothingness. This notion that printing carries ubiquitousness with it is spread throughout Hugo's poetics and concurs with Nerval's more insolated idea, developed in L'Imagier de Harlem that sees printing as a tool for knowledge as well as for deception. Though Nerval's text doesn't have the same far-reaching significance and impact as Hugo's novel, both writers thus belong to the first authors to ever enter the field of what we would later call mediology.

Wer die Vorliebe Hugos für mittelalterliche Architektur kennt, wird über die genaue Bedeutung der Aussage « Dieses wird jenes töten » nachdenken müssen, da dieser Ausdruck die Literatur der Architektur radikal entgegensetzt. Hugo, der die Architektur eher als Symbol für Sprache denn als Baukunst betrachtet, richtet sein Augenmerk auf das literarische Medium, dessen Variationen sich auf die Autonomie und das Wesen der übermittelten Botschaft auswirken: Mit dem Übergang von Bauten zu Büchern geht man also von einer eindeutigen zu einer mehrdeutigen 
Aussage über, die beispielsweise mal Unsterblichkeit, mal Aussterben bedeuten mag. Die gleiche Überzeugung von der Allgegenwärtigkeit des Buchdrucks, die sich in der gesamten Dichtung von Hugo erkennen lässt, findet man sporadisch auch bei Nerval, der in L'Imagier de Harlem den Buchdruck als ein Mittel sieht, das sowohl der Erkenntnis als auch dem Irrtum zu Diensten stehen kann. Selbst wenn die Schriften Nervals von keiner so weittragenden Bedeutung wie die von Hugo sind, zählen beide Autoren zu den ersten Erfindern der später so genannten Mediologie.

\section{AUTEUR}

\section{MAX MILNER (†)}

Max Milner, professeur émérite de littérature française à l'université Paris-III Sorbonne nouvelle, fut pendant plus de vingt-cinq ans président de la Société des études romantiques et dixneuviémistes. Spécialiste de la littérature romantique et de Saint-Jean de la Croix (Poésie et vie mystique chez saint Jean de la Croix, 1951 ; Le Diable dans la littérature française de Cazotte à Baudelaire, 1772-1861, 1960 ; Baudelaire : enfer ou ciel, qu'importe !, 1967), ses travaux ont également en grande partie porté sur les enjeux de la vision et de la fantasmagorie dans l'imaginaire littéraire ( $L a$ Fantasmagorie : essai sur l'optique fantastique, 1982 ; L'imaginaire des drogues : de Thomas de Quincey à Henri Michaux, 2000 ; L'Envers du visible, 2005). 